\title{
Knowledge of Gestational Diabetes Mellitus among Prenatal Women Attending a Public Health Center in Al-Khobar, Saudi Arabia
}

\author{
Abdullah Alnaim
}

Family and Community Medicine Department, College of Medicine-Al Hassa, King Faisal University, AI Hfouf, Kingdom of Saudi Arabia

\begin{abstract}
Background: Gestational diabetes mellitus (GDM) is a current health problem that affects pregnant women and fetuses. Addressing GDM offers a window of opportunity for the prevention of DM in Saudi Arabia. Here, we aimed to assess the current level of knowledge regarding GDM among prenatal women attending primary healthcare centers (PHCs) in the city of Al-Khobar. We also aimed to increase the awareness of GDM and to determine the significance of this study within the Saudi Arabian context.

Methods: After obtaining informed consent from the study subjects, a pre-validated, structured questionnaire was used to assess GDM knowledge among prenatal women attending PHCs in the city of Al-Khobar.

Results: We included 471 prenatal women in the study (mean age: 29 years). Overall, 36.5\% of the women had good knowledge of GDM, while 50.5\% had fair knowledge, and 13\% had poor knowledge. Most women (> 99\%) were literate and identified themselves as housewives (75.4\%). The knowledge score concerning how GDM is diagnosed was $45.3 \%$. Approximately $41.4 \%$ women had undergone tests for GDM. Furthermore, $65.8 \%$ of the participants were aware of GDM treatment. However, only 39.5\% were aware of the consequences of GDM. A major source of GDM knowledge was reported to be from friends and relatives.

Conclusion: Although knowledge of GDM as a disease in Al-Khobar community was average, the depth of this knowledge was poor. Thus, doctors and healthcare providers must play a greater role in improving health education among prenatal women. Furthermore, suitable intervention programs should urgently be undertaken by the Ministry of Health to improve GDM knowledge among this population.
\end{abstract}

Keywords: gestational diabetes, knowledge, level of knowledge, awareness.

\section{INTRODUCTION}

Gestational diabetes mellitus (GDM) is defined as any degree of glucose intolerance with onset or first recognition occurring during pregnancy ${ }^{(1)}$. According to the World Health Organization (WHO), gestational diabetes can be defined as hyperglycemia with onset or first identification occurring during pregnancy ${ }^{(2)}$.

Globally, the prevalence of GDM is approximately $7 \%$, but the prevalence ranges from $1 \%$ to $14 \%$ of all pregnancies in some countries depending on the population studied and the diagnostic tests employed (1). Some countries have higher prevalence rates (19\%) than others ${ }^{(3)}$. For example, recent studies have revealed higher prevalences of gestational diabetes in Norway and in the United Arab Emirates (UAE) $(37.7 \%)$ than in Mexico $(30.1 \%)^{(4,5)}$. The prevalence of GDM in the Kingdom of Saudi Arabia ranges from $8.9 \%$ to $12.5 \%$ based on a study reported in $2000^{(6)}$.

Risk assessment for GDM should be conducted upon the first prenatal visit. Clinical characteristics suggestive of a high risk of GDM include both modifiable factors, such as marked obesity and lifestyle factors, and non-modifiable factors, such as a personal history of GDM, glycosuria, or a strong family history of diabetes ${ }^{(1,8,9)}$.

GDM is associated with significant complications for both the mother and the fetus or newborn. Maternal risk includes an increased chance of requiring a
Cesarean section and higher risks of ketonemia, preeclampsia, and urinary tract infection. Neonatal risks include increased perinatal morbidity (e.g., macrosomia, neonatal hypoglycemia, and neonatal jaundice) and an increased risk of mortality. Women with a history of GDM have a higher risk of subsequent type 2 diabetes. Therefore, diagnostic testing and prevention measures should be undertaken during postnatal follow-up ${ }^{(1,7)}$. The prevalence of gestational diabetes has been steadily increasing in the Kingdom of Saudi Arabia. Therefore, we performed a crosssectional study to assess knowledge of GDM among prenatal women attending PHCs in hospitals located in the city of Al-Khobar.

\section{Definition of Terms}

- Gestational diabetes mellitus: any degree of glucose intoler- ance with onset or first recognition during pregnancy ${ }^{(1)}$.

- Knowledge: The fact or condition of being aware of something (merriam-webster.com).

- Awareness: The ability to directly know and perceive, feel, or be aware of events. More

- broadly, it is the state or quality of being conscious of something.

- Level of knowledge: A higher score indicated better GDM knowledge. The maximum score was

This article is an open access article distributed under the terms and conditions of the Creative Commons Attribution (CC BY-SA) license (http://creativecommons.org/licenses/by/4.0/) 
25 and the minimum was 0 . The total knowledge score was categorized as follows: $0-8=$ poor knowledge, 9-16 = fair knowledge, and 17-25 = good knowledge ${ }^{(7)}$. The maximum score of GDM knowledge was calculated as 25 points.

\section{Literature review}

Different types of studies have been conducted throughout the world to identify and measure knowledge and awareness levels of GDM. Many studies have been conducted in various parts of Saudi Arabia to contribute to the health promotion of GDM awareness among pregnant women. However, to the best of our knowledge, this is the first study that has been conducted in primary healthcare centers in AlKhobar region.

The main objectives of this study were to measure knowledge of GDM, including risk factors, importance of screening, and postpartum diabetes, among pregnant women in Al-Khobar region, as well as to determine the main source of such GDM knowledge.

Recent clinical studies have reported the prevalence of GDM among women to be $8.2 \%$ in Jazan, Saudi Arabia and $5.1 \%$ in Yemen ${ }^{(10,11)}$.

Many studies have reported knowledge or awareness scores for GDM among pregnant or the general population. In the United Arab Emirates (Sharjah), $73.5 \%$ of individuals were aware of the condition. In India, $56.7 \%$ of women were reported to have fair knowledge, while another study in India reported average knowledge scores ${ }^{(12,13,14)}$. Literature reviews concerning the risk factors of GDM have found obesity to be the main risk factor, followed by a previous history of GDM, a family history of DM, older maternal age and hypertension $(10,11,15)$. Many individuals are unaware of the consequences of GDM during and after pregnancy, and clinical studies have shown different scores concerning awareness of its consequences.

A previous study reported that $75 \%$ of women were aware that GDM could cause complications during pregnancy, while $77 \%$ were aware that both the mother and the fetus could be affected. Additionally, $42 \%$ of women were aware that the children of mothers diagnosed with GDM during pregnancy were at a higher risk of developing diabetes mellitus during their adulthood ${ }^{(16,15)}$. A recent study revealed that women with GDM had a 10-fold higher risk of developing type 2 diabetes mellitus during a 10-year follow-up period compared to women without $\operatorname{GDM}^{(18,14,22)}$.

In contrast, clinical trials have shown that women with GDM are at an increased risk of experiencing cardiovascular events compared to those without GDM and much of this risk is attributable to the development of type 2 diabetes ${ }^{(19)}$. Furthermore, a study concerning the relationship between subclinical thyroid disease and the incidence of GDM revealed a relationship between subclinical hypothyroidism and diabetes during pregnancy ${ }^{(23)}$. In a trial involving exercise for GDM, it was reported that women with
GDM should do both aerobic and resistance exercises at a moderate intensity a minimum of three times per week for 30-60 minutes ${ }^{(24)}$.

Another study investigating the dietary awareness of Saudi women with regard to GDM revealed that GDM patients require dietary counseling with nutritionists who should suggest special dietary strategies ${ }^{(25)}$. With regard to investigations concerning the source of information about (GDM), a UAE (Sharjah) study revealed that family members were a major source of awareness, while a study conducted in India revealed that television/radio was a major source $(12,13)$. Knowledge is an important component of health literacy, and studies have shown that inadequate knowledge about a disease leads to a poor understanding of medical information. This then leads to limited adherence to management plans and ultimately unfavorable pregnancy outcomes.

\section{METHODOLOGY}

- Study setting and time: This study was conducted in a PHC at the Ministry of Health Clinic, Al-Khobar, Saudi Arabia between 2019 and 2020.

- Study subject (inclusion criteria):

- Saudi nationality

- Currently pregnant

- Study design: Analytic cross-sectional study conducted via a self-administered questionnaire, which was obtained from the original author who had previously published a study that validated this questionnaire ${ }^{(26)}$.

- Sample size: The sample size was calculated using the website RAOSOFT. Correction factor: $5 \%$. Confidence interval: $95 \%$. Total target population: 533. The sample size was calculated as 224 and was multiplied by 2 for a design effect $=471$.

- Study variables: Dependent variable: Knowledge of GDM. Independent variable: Sociodemographic data of the participants (e.g., age, education status, occupation, gestational age, family history, and history of disease).

- Sample selection: Simple random sampling.

- Data collection methods: Data were collected through a self-administered questionnaire ${ }^{(26)}$ that was distributed to the women who visited the Prenatal Care Clinic.

\section{Data management and analysis plan:}

After answering the questionnaire, the forms were collected, and any form with less than $50 \%$ of the questions being answered was excluded from the analysis. All of the collected data were entered into Microsoft Excel and double-checked by the principle investigator. Subsequently, the data were transferred to SPSS version IBM® SPSS $®$ Statistics 23.0. Following this, a frequency table was produced with percentages, measures of central tendency, and dispersion to explore the association between the predictor and dependent (outcome) using chi-squared tests, 
ANOVA, correlations, t-tests, and regression analysis for comparing variables.

Ethical considerations:

Approval was given by the IRB prior to the initiation of the study. Approval was also given by the General Directorate of Health in the Eastern province, Ministry of Health before the study was conducted.

All information collected from the questionnaire were kept confidential, and the participants were informed verbally that their involvement was voluntary and that completing the distributed questionnaires implied that they had agreed to take part in the study. Informed consent was received from the participants before their inclusion in the study. Participants could refuse to participate in the study.

Funding: No budget, self-funded.

\section{RESULTS}

The study included 471 pregnant women attending for prenatal care at a PHC. The participants aged between 17 and 49 years (mean age: 29 years). Less than $1 \%$ of the participants were illiterate, and $39.3 \%$ had a high level of education. Most of the women identified themselves as housewives $(75.4 \%)$, and only $24.6 \%$ were employed. Of the study participants, $68.6 \%$ had children, while $31.4 \%$ did not. Of the pregnant participants, $35.7 \%$ were in their first trimester, $29.7 \%$ in their second trimester, and $34.6 \%$ in their third trimester (Table 1). Less than half of the participants $(46.1 \%)$ had a family history of diabetes mellitus. Only $9.1 \%$ had a family history of GDM, and $32.5 \%$ had a history of hypertension (HTN). A family history of hypothyroidism and cardiovascular disease (CVD) was noted only in $6.8 \%$ and $15.1 \%$ of participants, respectively (Table 2).

With regard to personal history of disease, $97 \%$ of the participants never had diabetes mellitus, $94.1 \%$ did not have GDM and $95.1 \%$ did not have HTN. Furthermore, hypothyroidism, POS, and obesity were not reported in $96.6 \%, 98.5 \%$, and $91.7 \%$ of participants, respectively (Table 3 ).

With regard to overall knowledge, $77.7 \%$ were aware of the meaning of diabetes, and $77.9 \%$ understood the function of insulin. An awareness of the chronicity of diabetes was found in $67.9 \%$ of participants. Moreover, $73.7 \%$ believed that diabetes mellitus is a genetic disease, and $47.3 \%$ believed the GDM could occur again during future pregnancies if it had occurred previously. However, when asked about owning a blood sugar meter and their own sugar levels (based on guidelines) ${ }^{(1)}$, only $40.3 \%$ owned a glucometer, and $76.4 \%$ had previously undergone blood sugar level testing (Table 4).

When assessing the awareness of the risk factors for GDM, 76.2\% believed that obesity increased the risk of diabetes and $74.5 \%$ believed that stress was a risk factor. Additionally, $78.8 \%$ were aware that regular exercise could control diabetes mellitus, and $78.3 \%$ believed that unhealthy foods increased the risk of GDM. Only $32.7 \%$ were aware of the relationship between HTN and GDM (Table 5).

Table (5) presented the results concerning the awareness of GDM complications. In all, 51.8\% of the participants believed that increased fetal birth weight was a complication and $72.6 \%$ believed that the possibility of developing diabetes mellitus in the future was a complication. Only $40.8 \%$ believed that an increased risk of congenital anomalies could be a complication in cases of undiagnosed diabetes mellitus, and $47.6 \%$ did not believe that terminating a pregnancy was required in cases of uncontrolled GDM. Only $35.5 \%$ of the participants were aware of the future childhood effects on the infants of GDM mothers. In all, $57.7 \%$ of the participants were aware of the risk of developing diabetes mellitus in the future as a consequence of GDM. Only $48.2 \%$ of all participants believed that breastfeeding has an effect on improving glucose metabolism. Of the participants, $41.6 \%$ had received preconception GDM counseling (Table 5).

Knowledge of when GDM was typically diagnosed varied, with $60.9 \%$ answering the first trimester, $8.3 \%$ the third trimester, and $30.8 \%$ the second trimester (Figure 1).

When asked about diagnostic modality, 15.3\% answered urine test, $40.8 \%$ answered blood test, and 43.9\% answered OGTT (Figure 2).

Overall, $19.5 \%$ believed that only diet and exercise were required to treat GDM, 5.3\% believed that oral hypoglycemic agents were required, $6.6 \%$ suggested the use of insulin and $68.6 \%$ believed that all of the above measures were required based on the level of control of GDM (based on guidelines) that was needed (Figure 3). Of the participants, $41.6 \%$ had received preconception GDM counseling (Table 5).

Participant awareness of GDM being a risk factor during pregnancy and delivery is shown in figure (4).

As reported, shoulder dystocia, C-S, diabetic ketoacidosis, intrauterine fetal death, and postpartum hemorrhage were reported by $5.9 \%, 21 \%, 14.9 \%$, $18.3 \%$, and $7.6 \%$ of participants, respectively as possible consequences of GDM, and $39.5 \%$ believed that all these could be consequences of GDM. The major reported source of knowledge of GDM was friends and relatives (33.8\%), and only $14.9 \%$ reported their source of knowledge as their healthcare provider (Figure 5). Overall, the average knowledge score was $14.44 \pm 5.104$. $13 \%$ of participants had poor knowledge, $50.5 \%$ had fair knowledge and $36.5 \%$ had good knowledge of GDM (Figure 6). Knowledge scores increased with education level and age. 
Table (1): Demographic profile of the study participants

\begin{tabular}{lll} 
Characteristics & $\mathbf{N}=\mathbf{4 7 1}$ & $\mathbf{\%}$ \\
\hline Age & $\mathbf{4 7 1}$ & \\
Min-Max & $17-49$ & \\
Mean \pm SD & $29.52 \pm 6.395$ & \\
Education level & & \\
Illiterate & 4 & 0.8 \\
Primary school & 17 & 3.8 \\
Intermediate school & 40 & 8.5 \\
Secondary school & 225 & 47.8 \\
Advanced & 185 & 39.3 \\
Occupation & & \\
Housewife & 355 & 75.4 \\
Employee & 116 & 24.6 \\
Parity & & \\
No children & 148 & 31.4 \\
One or more & 323 & 68.6 \\
& & \\
GA & & \\
& & 35.7 \\
$1^{\text {st }}$ trimester & 168 & 29.7 \\
$2^{\text {nd }}$ trimester & 140 & 34.6 \\
$3^{\text {rd }}$ trimester & 163 & \\
\hline GA: Gestational age; SD: Standard deviation &
\end{tabular}

Table (2): Family history of the study participants

\begin{tabular}{lcr}
\hline Disease & $\mathbf{N}=\mathbf{4 7 1}$ & $\boldsymbol{\%}$ \\
\hline DM & 217 & 46.1 \\
GDM & 43 & 9.1 \\
HTN & 153 & 32.5 \\
HT_H & 32 & 6.8 \\
CVD & 71 & 15.1
\end{tabular}

DM: Diabetes mellitus; GDM: Gestational diabetes mellitus; HTN: Hypertension; HT_H:

Hypothyroidism; CVD: Cardiovascular disease

Table (3): History of disease of the study participants

\begin{tabular}{llc}
\hline Disease & $\mathbf{N}=\mathbf{4 7 1}$ & $\boldsymbol{\%}$ \\
\hline DM & 14 & 3 \\
GDM & 28 & 5.9 \\
HTN & 23 & 4.9 \\
HT_H & 16 & 3.4 \\
PCOS & 7 & 1.5 \\
Obesity & 39 & 8.3
\end{tabular}

DM: Diabetes mellitus; GDM: Gestational diabetes mellitus; HTN: Hypertension; HT_H: Hypothyroidism; PCOS:

Polycystic ovary syndrome; N: number of participants. 
Table (4): Knowledge of GDM

Questions answered "yes" Number of participants who answered "yes"

$\mathrm{N}=471$

People with diabetes have higher sugar levels in their blood.

$\%$

Insulin is a hormone that decreases sugar levels.

77.7

77.9

Diabetes is a disease that cannot be cured but can be controlled.

67.9

Do you think a family history of diabetes has an effect on developing

diabetes?

If you have had gestational diabetes during one pregnancy, will it occur

again in future pregnancies?

47.3

Do you own a blood sugar meter (glucometer)?

40.6

Have you ever tested your blood sugar before?

76.4

Table (5): Awareness of GDM risk factors and complications.

\begin{tabular}{|c|c|c|}
\hline \multicolumn{3}{|c|}{ Number of participants who answered "yes" } \\
\hline Risk factor & & \\
\hline Obesity/overweight & 359 & 76.2 \\
\hline Stress/depression & 351 & 74.5 \\
\hline Exercise & 371 & 78.8 \\
\hline Unhealthy foods & 369 & 78.3 \\
\hline HTN & 154 & 3 \\
\hline \multicolumn{3}{|l|}{ Complication } \\
\hline Increased fetal birth weight & 244 & 51.8 \\
\hline Possibility of developing DM & 342 & 72.6 \\
\hline $\begin{array}{l}\text { Increased risk of congenital anomalies in cases } \\
\text { of undiagnosed DM }\end{array}$ & 192 & 40.8 \\
\hline $\begin{array}{l}\text { No potential need to terminate pregnancy } \\
\text { in cases of uncontrolled GDM }\end{array}$ & 224 & 47.6 \\
\hline $\begin{array}{l}\text { Future effects on the infants of GDM mothers during } \\
\text { childhood }\end{array}$ & 167 & 35.5 \\
\hline Future DM as a consequence of GDM & 272 & 57.7 \\
\hline Breastfeeding can improve glucose metabolism & 227 & 48.2 \\
\hline Preconception counseling about GDM & 196 & 41.6 \\
\hline
\end{tabular}


https://ejhm.journals.ekb.eg/

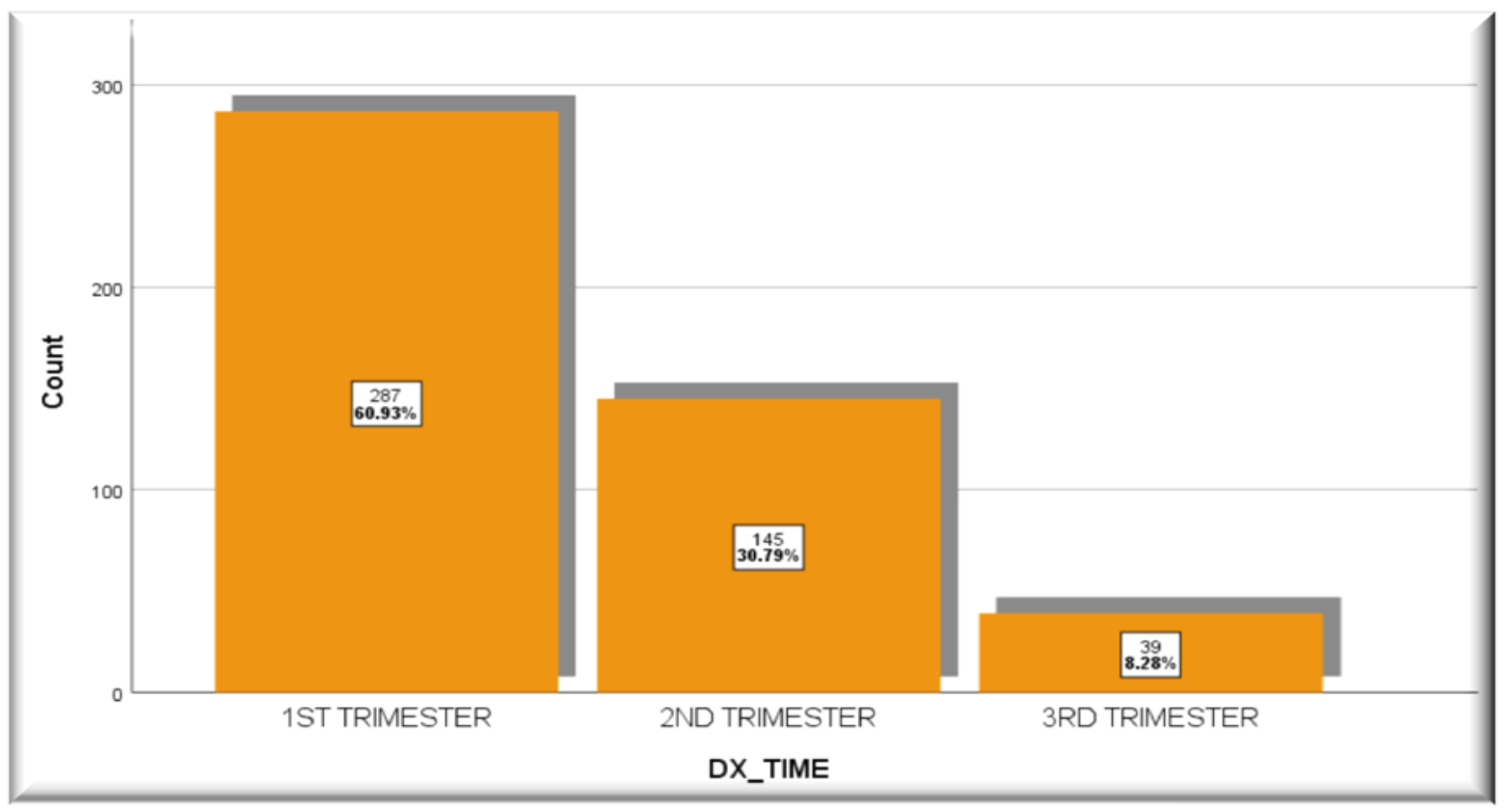

Figure (1): Participant responses of the time at which GDM is typically diagnosed.

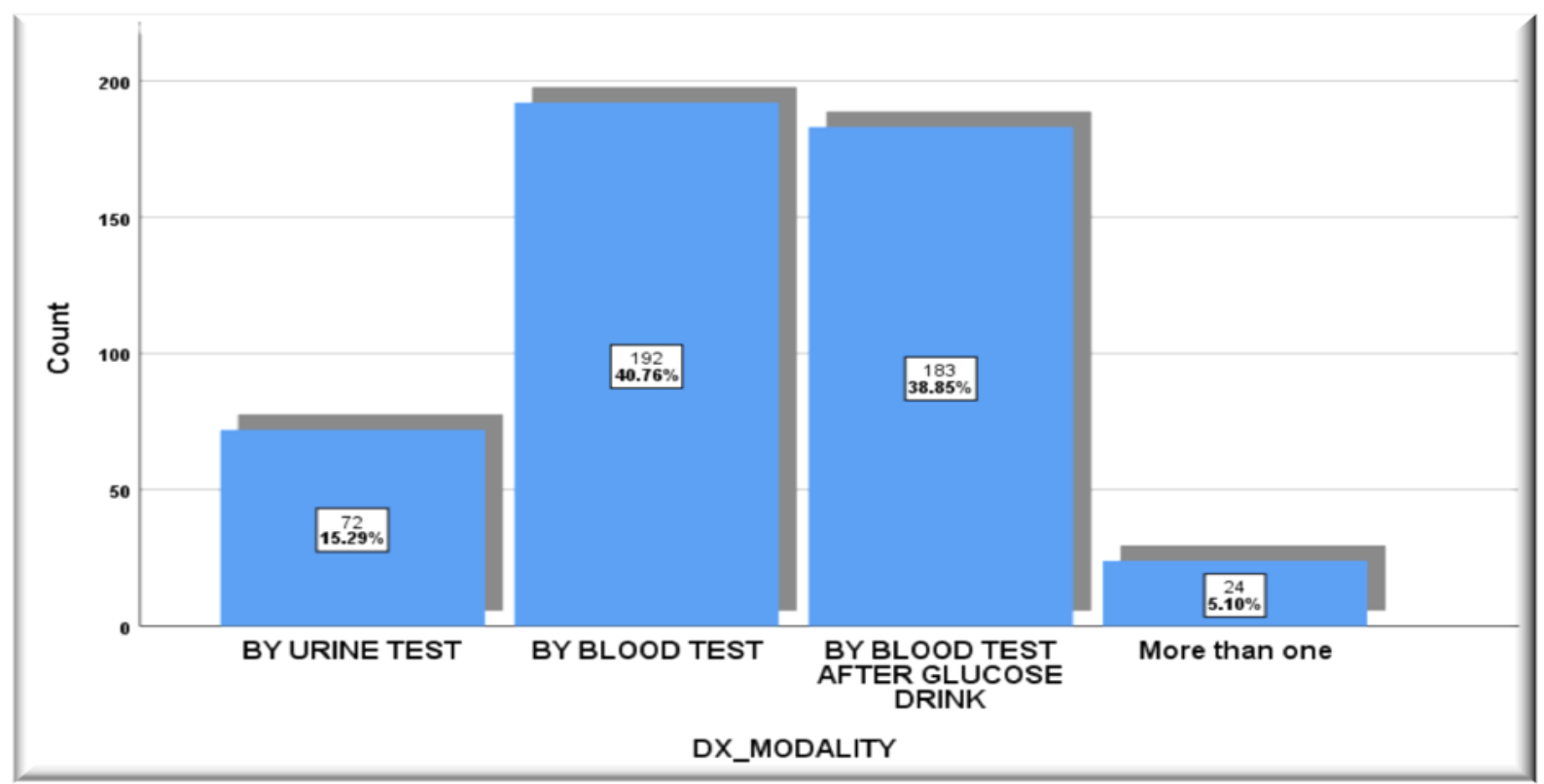

Figure (2): Participant responses of the diagnostic modality of GDM 


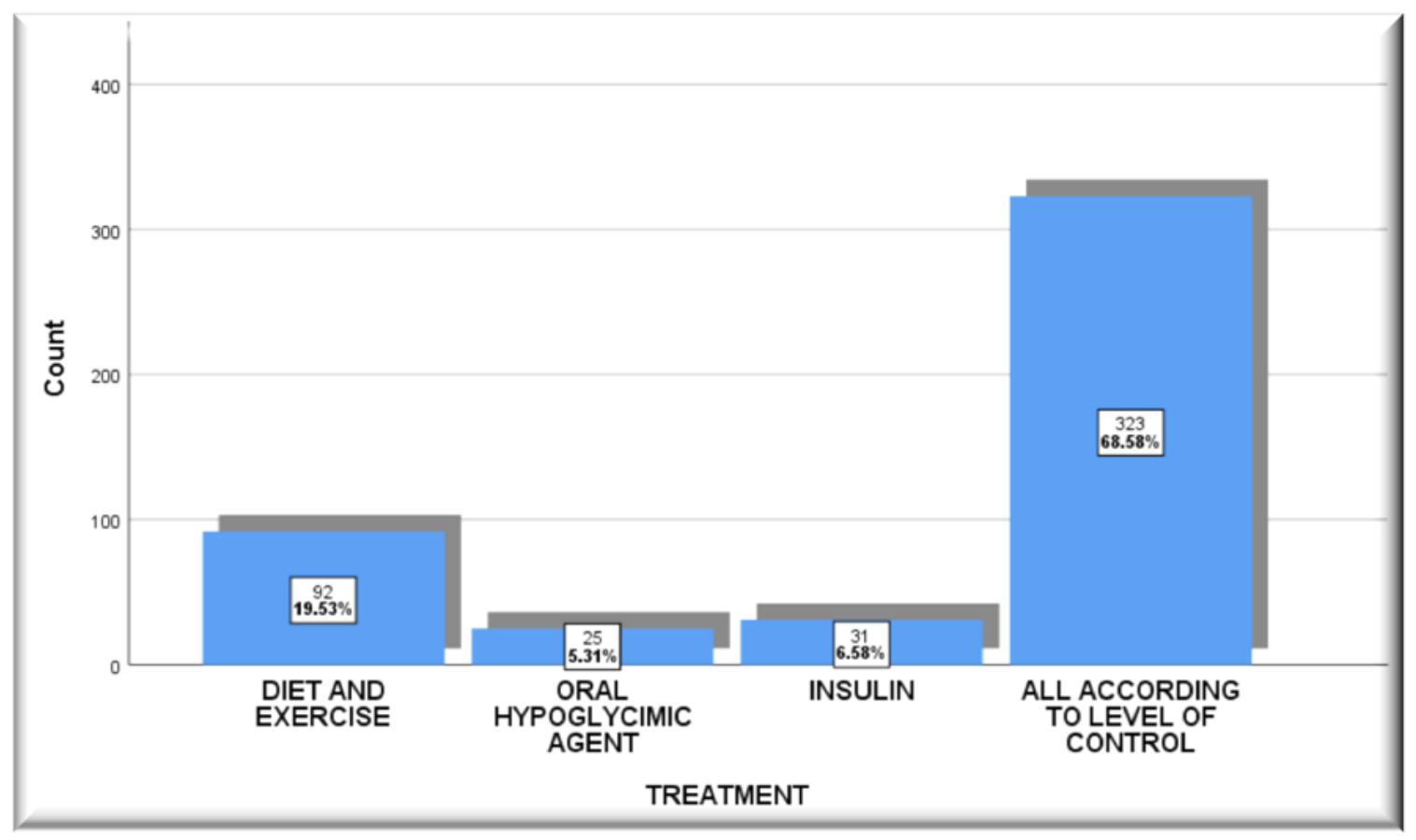

Figure (3): Participant responses of GDM treatment types

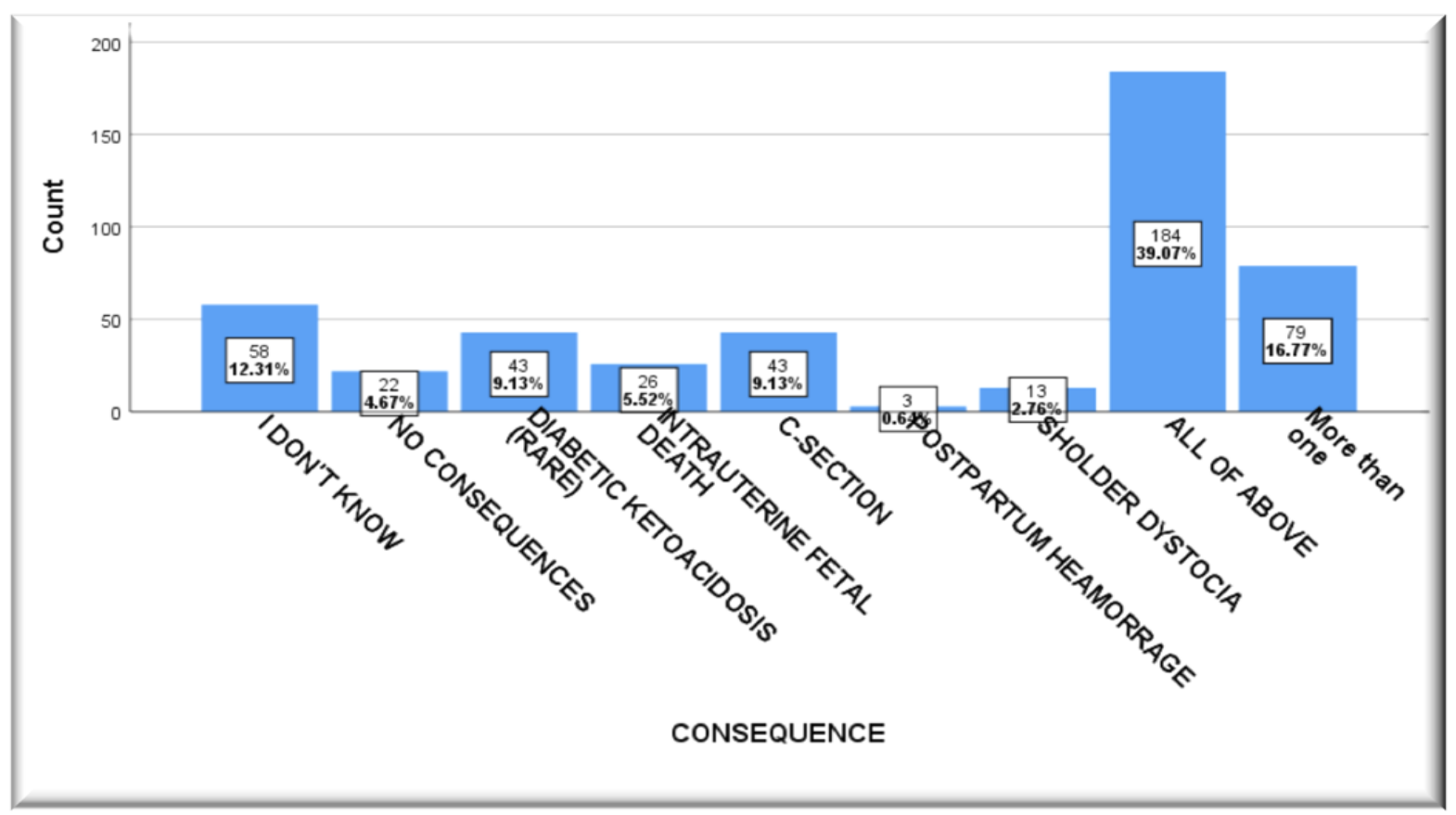

Figure (3): Participant responses of the consequences of GDM 


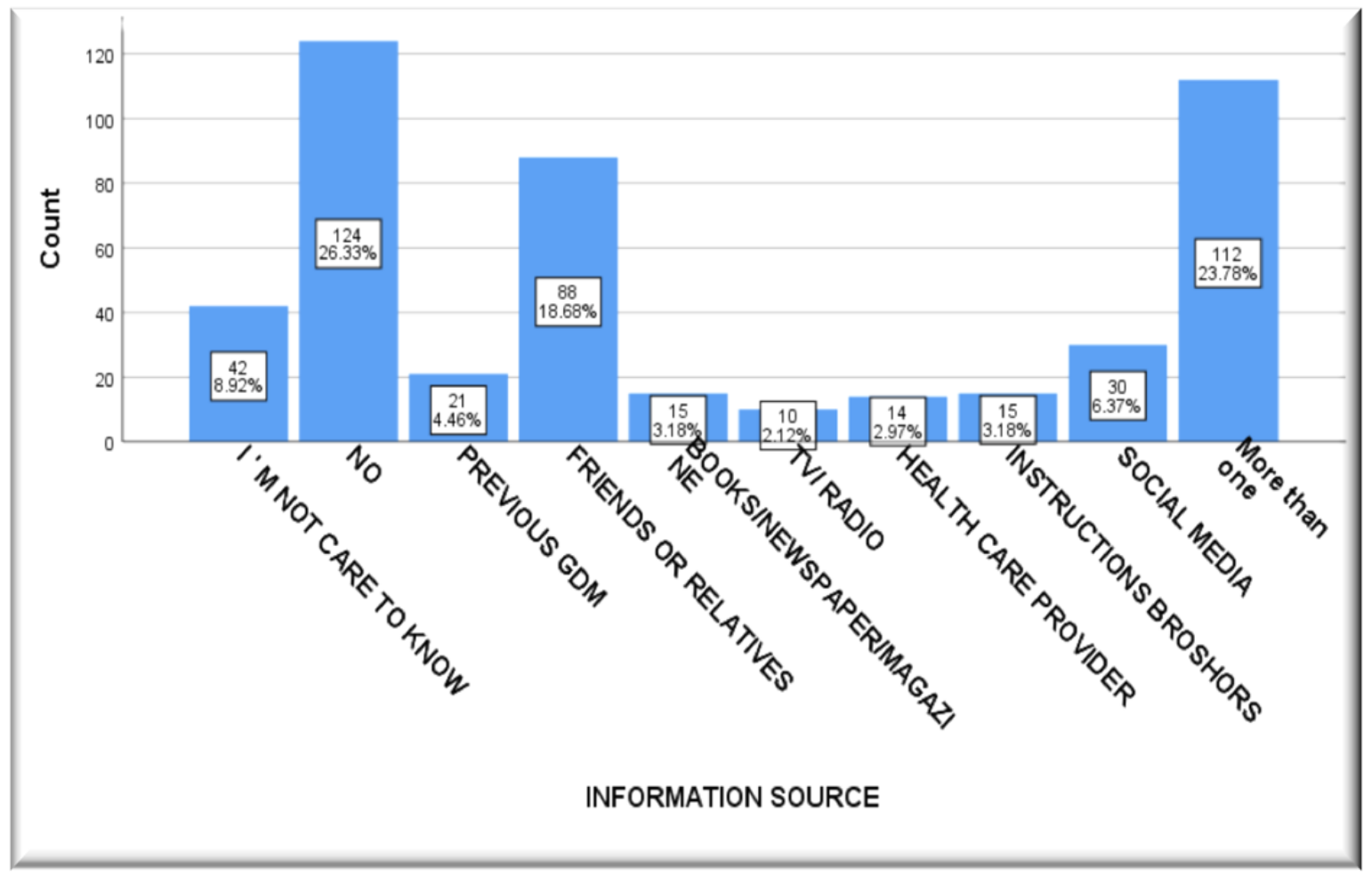

Figure (4): Sources of GDM knowledge

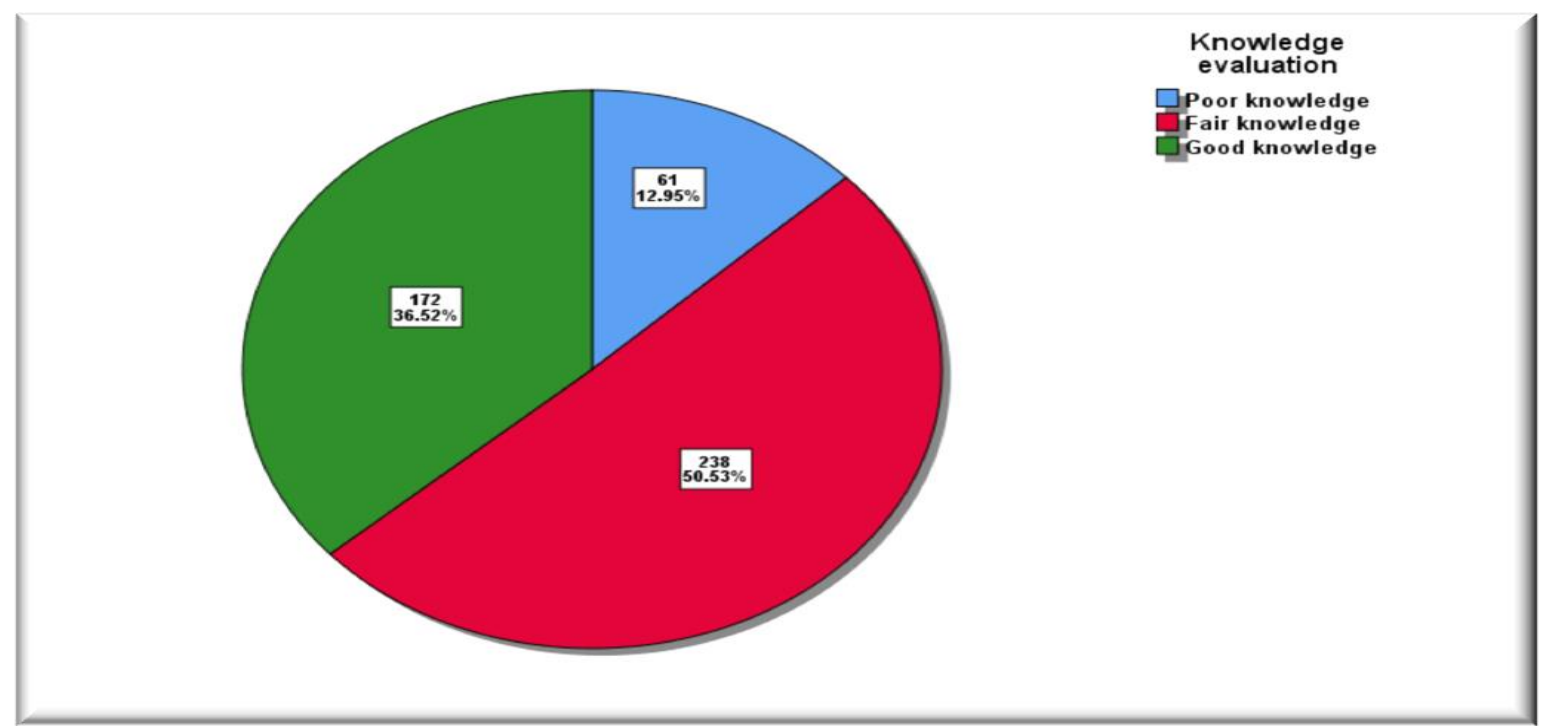

Figure (5): GDM knowledge scores among pregnant women

\section{DISCUSSION}

Preventing an epidemic of diabetes involves detecting it during pregnancy. Therefore, it is important that pregnant women are aware of GDM during pregnancy and understand its consequences. There is emerging evidence that GDM education and awareness provided by PHCs, along with an increased motivation for self-care, can improve GDM care and reduce its complications. Our study showed that only $36.5 \%$ of participants had good knowledge about GDM, while most participants $(50.5 \%)$ had fair knowledge, and $13 \%$ had poor knowledge. These findings are similar to those in previous international studies $(12,13,14,20)$. In a study conducted in Southern India, only $17.5 \%$ of participants had good knowledge,
$56.7 \%$ had fair knowledge and $25.8 \%$ had poor knowledge. An important difference between our study and this study was that their sample size was much smaller at only 120 prenatal women ${ }^{(13)}$. In the current study, ANOVA testing revealed a relationship between age and the level of GDM knowledge (mean $=26.23,29.14$ and 31.16, respectively). Furthermore, chi-square testing revealed higher knowledge scores with increased education levels. However, another study conducted in Sharjah reported no relationship between age, education, and level of awareness ${ }^{(12)}$.

Another study revealed that awareness was greater in young females aged $<40$ years, those residing in urban areas and those who were educated (20). Our participants had good knowledge about 
general DM and GDM and its risk factors. Although the majority of participants believed that they should undergo screening for GDM during pregnancy. The timing of such screening and the relevant diagnostic modality were unclear. In all, $39.1 \%$ were aware of the screening time of GDM (based on guidelines), and $41.4 \%$ knew the diagnostic modality used for GDM.

Many participants were unaware of the consequences of GDM during delivery and after pregnancy and the increased risk of developing type 2 diabetes mellitus in the future. Moreover, many participants were unaware of the harmful effects of hypertension in patients with GDM. In fact, having both chronic hypertension and gestational diabetes during pregnancy can often lead to poor pregnancy and perinatal outcomes, represented by increased maternal, obstetrical and neonatal morbidity ${ }^{(21)}$.

Pregnant women are recommended and encouraged to breastfeed their babies, and they are informed of this advice from their first prenatal care visit. Despite its well-recognized benefits to the health outcomes of both mother and child, the rate of breastfeeding is lower in women with GDM (17). Unfortunately, our study revealed indifference to breastfeeding and its importance in the treatment of postpartum GDM. The PHC did not play an effective role in the promotion and education of community knowledge of GDM, as suggested by the results concerning information sources, with $64.8 \%$ of participants being aware of GDM but only $14.4 \%$ reporting receiving information from healthcare providers. Overall, the main source of information was reported to be friends and relatives, which is similar to findings reported in other studies ${ }^{(12)}$.

The strength of our study was that the data were collected from a primary healthcare unit. Moreover, the present study is the first to measure GDM knowledge in a PHC in the city of Al-Khobar. The sample size $(n=471)$ was large, and the population was diverse. A limitation of the study was that it was conducted in only one region in Saudi Arabia. The cross-sectional nature of the study is also a limitation.

\section{CONCLUSION AND RECOMMENDATIONS}

Overall, the knowledge of GDM among pregnant women was found to be average. Therefore, health education programs about GDM should be conducted to improve the knowledge of pregnant women to ensure better GDM care. Furthermore, physicians, nurses, and diabetes educators should play an active role to ensure that GDM is discussed as a serious illness and not just a transient condition in pregnant women. It is encouraging to see the role played by mass media in spreading information about GDM and other diseases.

\section{Conflict of interest}

No potential conflicts of interest relevant to this article are reported.

\section{Acknowledgments:}

I would like to thank my wife, Dr. Latifah Alnaim, and the nursing staff at the primary healthcare center in Al-Khobar for their help. I would also like to thank the women who provided their valuable time and participated in the study by answering the questionnaire. Disclaimer: This research was submitted in partial
fulfillment of the Saudi Board in Family Medicine.

\section{REFERENCES}

1. American diabetes association(2003): Gestational Diabetes Mellitus. Diabetes Care, 26 (1): s103-s105.

2. World Health Organization (1999): Definition, Diagnosis and Classification of Diabetes Mellitus and its Complications. Report of a WHO consultation. Part 1: Diagnosis and Classification of Diabetes Mellitus. Geneva. https://apps.who.int/iris/handle/10665/66040

3. Schmidt MI, Duncan BB, Reichelt AJ, Branchtein L, Matos MC et al. (2001): Gestational Diabetes Mellitus Diagnosed with a 2-h 75-g Glucose Tolerance Test and Adverse Pregnancy Outcomes. Diabetes Care, 24: 11511155 .

4. Jenum AK, Morkrid K, Sletner L (2012): Impact of Ethnicity on Gestational Diabetes Mellitus with the WHO and the Modified International Association of Diabetes and Pregnancy Study Groups Criteria: A Population-based Cohort Study. Eur J Endocrinol., 166: 317-324. 9.

5. Agarwal MM, Dhatt GS, Shah SM (2010): Gestational Diabetes Mellitus Simplifying the International Association of Diabetes and Pregnancy Diagnostic Algorithm using Fasting Plasma Glucose. Diabetes Care, 33: 2018-2020.

6. Ardawi MS, Nasrat HA, Jamal HS, Al-Sagaaf HM, Mustafa BE (2000): Screening for Gestational Diabetes Mellitus in Pregnant Females. Saudi Med J., 21: 155160.

7. The American College of Obstetricians and Gynecologists, Committee (2011): Screening and Diagnosis of Gestational Diabetes Mellitus, Opinion Number 504 https://pubmed.ncbi.nlm.nih.gov/21860317/

8. American Diabetic Association (2004): Gestational Diabetes Mellitus. Diabetes Care, 27 (1): S88-90.

9. Zhang C, Ning Y (2011): Effect of Dietary and Lifestyle Factors on the Risk of Gestational Diabetes: Review of Epidemiologic Evidence. Am J Clin Nutr., 94: 1975S9S.

10. Amani O, Mohamed S, Mona A et al. (2017): Gestational Diabetes Prevalence and Risk Factors among Pregnant Women -Jazan Region, Saudi Arabia. https://doi.org/10.5603/DK.2017.0028

11. Abdullatif $\mathrm{D}$, amat al-Khaleq $\mathrm{O}$, abdulelah $\mathrm{H}$ et al. (2016): Prevalence and Risk Factors of Gestational Diabetes Mellitus in Yemen. International Journal of 
Women's Health. https://doi.org/10.2147/IJW H.S97502.

12. Amr E, Batool A (2017): Gestational diabetes awareness in women of childbearing age in Sharjah . Global Journal of Obesity, Diabetes and Metabolic Syndrome ,4: 51-53.

13. Sujindra E, Bupathy A (2016): What Do Mothers Know about Gestational Diabetes: Knowledge and Awareness? https://doi.org/10.18231/23942754.2016.0021.

14. Vanishree S, M. Anitha R, B. W. C. Sathiyasekaran et al. (2013): Awareness of Gestational Diabetes Mellitus among Antenatal Women in a Primary Health Center in South India. Indian Journal of Endocrinology and Metabolism, 17 (1): 146.

15. Eman A, Magda Y (2015): Gestational Diabetes among Saudi Women: Prevalence, Risk Factors and Pregnancy Outcomes. https://doi.org/10.5144/02 564947.2015.222

16. Deepa A, Vanshika G (2015): Awareness of Gestational Diabetes among Young Nulliparous Literate Women in Urban Areas in Mumbai, India. International Journal of Therapies and Rehabilitation Research, 4 (5): 276.

17. Much D, Andreas B, Michaela $R$ et al. (2014): Beneficial Effects of Breastfeeding in Women with Gestational Diabetes Mellitus. Molecular Metabolism, 3 (3): 284-92.

18. Himali H, Rasika H, Rajitha W (2017): Gestational Diabetes Mellitus and Risk of Type 2 Diabetes 10 Years after the Index Pregnancy in Sri Lankan Women-A Community Based Retrospective Cohort Study. Plos One, 12 (6): e0179647.
19. Baiju R S, Retnakakaran R, Gillian B (2008): Increased Risk of Cardiovascular Disease in Young Women Following Gestational Diabetes Mellitus. Diabetes Care, 31: 1668-69.

20. Praneet W, Vitull K, Meghna G et al. (2016) :Awareness about Gestational Diabetes Mellitus in Females among General Population. Journal of Clinical Diabetology 3: 2-5.

21. Ana C, Ana Catarina M , Ricardo $R$ et al. (2016): Chronic Hypertension with Gestational Diabetes Mellitus: What about Complications? Experimental and Clinical Research Original Article, 3 (4): 180-83.

22. Ghattu V, Jacqueline C. , Sargoor R et al. (2007): Gestational Diabetes and the Incidence of Diabetes in the 5 Years Following the Index Pregnancy in South Indian Women. Diabetes Research and Clinical Practice, 78 (3): 398-404.

23. Carmen M, Brian M, Donald D et al. (2012): Relationship of Subclinical Thyroid Disease to the Incidence of Gestational Diabetes. Obstetrics and Gynecology, 119 (5): 983-88.

24. Cliantha P, Jeff S C (2015): Exercise Guidelines for Gestational Diabetes Mellitus. https://doi.org/10.4239/wjd.v6.i8.1033.

25. Ismail E M, Mohamed S I (2013): Dietary Awareness of Saudi Women with Gestational Diabetes. Food and Public Health, 3 (6): 341-45.

26. Alnaeem L (2019): Awareness of Gestational Diabetes among Antenatal Women at The King Fahd Military Medical Complex Hospital in Dhahran, Saudi Arabia. Available at: https://ejhm. journals.ekb.eg/article_32977_b86b1801a7809aff53d3e 48ebdbe6aec.pdf. 